\title{
Exercícios terapêuticos nas desordens temporomandibulares: uma revisão de literatura
}

\section{Therapeutic exercises in temporomandibular disorders: a literature review}

\author{
Sâmia A mire M alufí, Bruno Gonçalves Dias M oreno², Patrícia Pereira A Ifredo30, \\ A mélia Pasqual M arques屯 , Graziela R odrigues ${ }^{5}$
}

Estudo desenvolvido no Fofito/ FMUSP - Depto. de Fisioterapia, Fonoaudiologia e Terapia Ocupacional da Faculdade de Medicina da Universidade de São Paulo, SP, Brasil

1 Fisioterapeuta do Movicet Movimento Centro de Estudos e Terapia, Campinas, SP

2 Fisioterapeuta do Depto. de Fisioterapia das Faculdades Adamantinenses Integradas, Adamantina, SP

3 Fisioterapeuta Ms.; doutoranda no Programa de Pós-Graduação em Fisiopatologia Experimental da FMUSP

${ }^{4}$ Profa. Dra. do Fofito/FMUSP

5 Fisioterapeuta do Movicet

ENDEREÇO PARA

CORRESPONDÊNCIA

Sâmia A. Maluf

Fofito/FMUSP

R. Cipotânea 51 Cidade

Universitária

05360-160 São Paulo SP

e-mail:

samia_maluf@uol.com.br

\section{A PRESENTAÇÃO \\ ago. 2008 \\ AcEITO PARA PUBLICAÇ̃̃o \\ nov. 2008}

Resum 0: A articulação temporomandibular faz parte do sistema estomatognático que, junto com os dentes, periodonto, coluna cervical, crânio e cintura escapular, é responsável pela mastigação, fonação, deglutição, respiração e expressão facial. Exercícios terapêuticos têm sido empregados na reabilitação e prevenção das disfunções temporomandibulares (DTM). Este estudo teve como objetivo revisar a literatura a respeito, verificando a eficácia dos exercícios terapêuticos nas DTM. Foram examinados periódicos do período entre 1991 e agosto de 2008, nas bases de dados Medline, Lilacs e Pubmed, utilizando as palavras-chave "desordem temporomandibular", "terapia por exercícios" e as correspondentes em inglês. Foram selecionados relatos de caso, artigos de revisão e ensaios clínicos com mais de 20 pacientes, num total de 53 artigos. A maioria relatou efeitos positivos na redução da dor, melhora da mobilidade e dos aspectos psicológicos, sugerindo que os exercícios podem contribuir no tratamento da DTM. Entretanto, o tipo, tempo de duração, número de repetições, freqüência e intensidade dos exercícios não está bem descrita. A falta de padronização das pesquisas, bem como da forma de avaliar, dificultam a comparação dos resultados. Mais estudos com métodos padronizados devem ser estimulados.

DescritoREs: Literatura de revisão como assunto; Terapia por exercício; Transtornos da articulação temporomandibular

A вSTRACT: The temporomandibular joint is part of the stomatognathic system, which comprises a complex set of orofacial structures, including teeth, cervical spine, cranium and shoulder. The system is responsible for masticatory, phonation, and deglutition functions, as well as for breathing and facial expression. Physical therapy exercises have been used for rehabilitation and prevention of temporomandibular disorders (TMD). The purpose of this study was to review studies on the subject and assess the effectiveness of physical therapy exercises for TMD. Case reports, review articles, and clinical trials with more than 20 patients, published from 1991 to mid-2008, were searched for in databases Medline, Lilacs and Pubmed, by using keywords "exercise therapy" "temporomandibular disorders", and the correspondent terms in Portuguese. Fifty-three studies were selected of which most showed positive effects on pain reduction, improvement in joint mobility and psychological aspects, suggesting that physical therapy exercises may be beneficial in TMD treating. However, the type, duration time, repetitions, frequency and intensity of the therapeutic exercises are not well described. The lack of research and assessment method standardization hinder result comparison. More studies with standardized methods must be stimulated.

KEY WORDS: Exercise therapy; Review literature as topic; Temporomandibular joint disorders 


\section{INTRODUÇÃO}

A articulação temporomandibular (ATM) é uma estrutura altamente especializada do complexo craniomandibular que está sujeita a comprometimentos de origem neurológica, ortopédica e musculoesquelética, originando as disfunções temporomandibulares (DTM) ${ }^{1}$, de etiologia multifatorial. Dificilmente os sinais e sintomas relacionados à ATM se apresentam de forma isolada ${ }^{2} \mathrm{e}$ incluem artromialgia facial (caracterizada por dor pré-auricular localizada na região da ATM), ruídos articulares durante a as funções da mandíbula, distúrbios nos movimentos mandibulares, fraqueza ou hiperatividade da musculatura mastigatória ${ }^{3}$.

A DTM pode ocorrer em todas as faixas etárias, mas sua incidência maior é entre 20 e 45 anos. Entre os 15 e 30 anos as causas mais freqüentes são as de origem muscular e, a partir de 40 anos, de origem articular. As mulheres são mais acometidas que homens em uma proporção de cinco para cada homem ${ }^{4}$.

Os exercícios terapêuticos têm sido muito empregados na reabilitação e prevenção da DTM, com o objetivo de aliviar a dor e melhorar a função, porém são escassos os trabalhos que comparem e discutam a eficácia dos mesmos. O objetivo deste estudo foi revisar os estudos da literatura e examinar a eficiência dos exercícios terapêuticos nas disfunções temporomandibulares.

\section{METODOLOGIA}

O levantamento bibliográfico foi feito nas bases de dados Medline, Lilacs e Pubmed, no período entre 1991 e meados de 2008. Foram selecionados artigos em português e inglês e utilizadas as palavras-chave "desordem temporomandibular", "terapia por exercícios" e as correspondentes em inglês. Os artigos que se referiam a outros tratamentos associados à prática de exercícios terapêuticos durante a reabilitação foram incluídos.

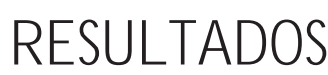

Foram selecionados 53 artigos sendo 12 de revisão, 5 estudos de caso e 36 ensaios clínicos. Destes, somente três utilizaram os exercícios terapêuticos sem associação a outros procedimentos e a maioria incluía uso de placa miorrelaxante e outros recursos fisioterapêuticos como eletroterapia, crioterapia, termoterapia, reeducação postural e proprioceptiva, terapia manual, mobilização e uso de aparelho para exercitar a mordida (BAE, bite assist exerciser). Os demais incluíam: orientação, relaxamento, exercícios domiciliares, medicação, terapia comportamental cognitiva, manipulação, treino da respiração e acupuntura.

As intervenções relatadas foram aplicadas em DTM miogênica (11 artigos), artrogênica (9), dor orofacial (4), deslocamento de disco sem redução (7), deslocamento de disco com redução (1 artigo). Em 21 artigos não havia informação sobre o tipo de DTM.

Como parâmetro de avaliação dos resultados, após o tratamento, a maioria dos artigos utilizou questionário próprio, outros os critérios diagnósticos para DTM (Research Diagnostic Criteria), alguns o inventário de ansiedade e o questionário McGill. Na avaliação da dor, além dos dados clínicos, foi utilizada palpação, escala visual analógica e dolorimetria; ainda, foi avaliada a amplitude de movimento. Nos exames complementares utilizaram-se eletromiografia, gravador de sons, axiografia computadorizada, ressonância magnética e imagem radiográfica.

Os artigos apontam efeitos positivos na redução da dor, na melhora da mobilidade, nos aspectos psicológicos e no quadro clínico, tais como melhora do sono, sintomas no ouvido, mastigação e fala. Apenas dois trabalhos apontaram ausência de efeito após o tratamento ${ }^{5,6}$, e três não relatam se houve melhora ${ }^{7-9}$. Os ganhos com o tratamento tendem a se manter longitudinalmente em curto e longo prazos ${ }^{10-16}$, sobretudo quando o paciente recebe orientações de autocuidado e treinamento para realizar exercícios no domicilio.

\section{DISCUSSÃO}

São comentados aqui inicialmente os artigos de revisão. Os estudos de caso e os ensaios clínicos serão examinados conforme a associação dos exercícios a outros recursos terapêuticos.

\section{E studos de revisão}

Os trabalhos de revisão ${ }^{9,10,17-19}$ relatam que a fisioterapia no tratamento da DTM está inserida entre as terapias de suporte, visando reduzir ou eliminar sinais e sintomas, mantendo ou recuperando a atividade funcional num menor espaço de tempo. Há uma forte relação entre DTM e alterações posturais, sendo o tratamento fisioterapêutico de extrema importância em tais disfunções e após intervenções cirúrgicas na $\mathrm{ATM}^{20}$. Os exercícios terapêuticos têm efeitos benéficos na melhora da dor e nas seqüelas da inatividade crônica do sistema musculoesquelético ${ }^{21}$.

Fricton $^{22}$ afirma que, nos estudos sobre DTM miogênica, os pacientes com DTM menos complexa podem ser tratados por orientações para autocuidado, exercícios e splints; os casos mais complexos devem ser tratados por uma equipe multidisciplinar.

McNeely et al. ${ }^{23}$ apontam a eficácia dos exercícios posturais associados a terapia manual e acupuntura na redução da dor e melhora da função e abertura bucal. Uma revisão sistemática ${ }^{18}$ analisou os estudos que avaliaram a eficácia de várias intervenções fisioterapêuticas na DTM, concluindo que os exercícios ativos, as mobilizações manuais, o treinamento postural em combinação com outras intervenções, a terapia a laser, os programas de retroalimentação (biofeedback), relaxamento e reeducação proprioceptiva podem ser mais eficazes do que o tratamento placebo ou que o uso de placas miorrelaxantes; e as combinações de exercícios ativos, terapia manual, correção postural e técnicas de relaxamento podem ser eficazes.

Bessa-Nogueira et al. ${ }^{24}$ avaliaram a qualidade metodológica de revisões sistemáticas, comparando o tratamento cirúrgico e não-cirúrgico da DTM, concluindo que alguns estudos não apontam diferença entre as abordagens e salientam que o mais preocupante é o aspecto ético dos ensaios clínicos, que não cumprem as normas internacionais de conduta, e os cuidados prestados aos doentes, que não se apoiavam em evidências científicas de alto nível. Mannheimer ${ }^{25}$ também conclui que as recomendações dos estudos 
devem ser vistas com precaução, pois a maioria não apresentam critérios claros de inclusão e exclusão nem resultados confiáveis.

\section{Exercício em associação com outros recursos ou terapias}

\section{A ssociado ao uso de placa}

O exercício terapêutico associado à placa miorrelaxante teve efeitos significantes no alívio da dor e na disfunção ${ }^{11,26}$; em alguns casos, somente o exercício terapêutico foi eficaz, podendo ser recomendado como primeira opção de tratamento na DTM miogênica $^{12,13,27-29}$. Grace et al. ${ }^{30}$ não observaram diferença entre os grupos tratados com terapias tradicionais, terapias tradicionais associadas à placa miorrelaxante e exercícios, nem tratamento por orientações associadas à placa.
Nos quadros de cefaléia associada à DTM, submetidos a tratamento durante dois anos com placa e exercícios, houve melhora na dor de cabeça: em pacientes com dor forte, a intensidade da dor passou a média e baixa e, mesmo, à ausência de dor ${ }^{5}$. Houve também diminuição da sensibilidade à palpação muscular ${ }^{8}$.

No caso de deslocamento anterior de disco, a associação de placa e exercícios terapêuticos apresentou resultados mais eficazes no reposicionamento do disco do que o tratamento só com placa de estabilização ou só com exercícios ${ }^{31}$. Stiesch-Scholz et al. ${ }^{32}$, por sua vez, obtiveram melhores resultados com placa e medicação do que com placa e fisioterapia; e Babadag et al. ${ }^{33}$ concluíram que o deslocamento anterior pode diminuir associando-se tratamento medicamentoso, exercícios mandibulares e placa miorrelaxante.

A melhora dos sintomas e função é clinicamente maior nos grupos tratados do que nos grupos sem tratamento, não havendo, porém, diferença entre as várias formas de procedimentos fisioterapêuticos ${ }^{34}$. A combinação de várias abordagens terapêuticas é mais eficaz que uma aplicada isoladamente ${ }^{1,35}$, embora Al-Ani et al. ${ }^{36}$ afirmem que o uso da placa miorrelaxante sozinha pode reduzir a dor ao repouso e à palpação.

\section{A ssociado à orientação de autocuidado e exercício domiciliar}

Alguns estudos referem que o uso das orientações deve ser associado a outros recursos ${ }^{12,14,15,37}$. Truelove et al. ${ }^{13}$ concluíram que a terapia com placa foi menos eficaz do que o tratamento conservador aliado a orientações de autocuidado; e Carlson et al. ${ }^{12}$ não encontraram diferença significativa entre pacientes tratados com placa, placa mais orientação, e exercícios domiciliares com orientação.

A repercussão das orientações de exercícios e reeducação postural nas

Quadro 1 Estudos em que se utilizou placa associada a exercícios e/ou outro procedimento

\begin{tabular}{|c|c|c|c|c|}
\hline Autoria, ano, tipo de estudo & $\mathrm{N}$ & Tratamento proposto & Disfunção & Resultados \\
\hline Felício et al (1991)26* & 33 & Exercícios & DTMM & Benéfico \\
\hline Widmark et al $(1994)^{7 * *}$ & 33 & Placa, injeções, medicação, terapia manual & DTMA & Indefinido \\
\hline Krogstad et al (1996) 8 & 103 & Orientações, exercícios & DOF & Indefinido \\
\hline Krogstad et al (1996) 57 & 103 & Orientações & DTM & Benéfico \\
\hline Krogstad et al (1998)28 & 16 & Exercícios & DTMM & Benéfico \\
\hline Magnusson \& Syren (1999)27 *** & 26 & Exercícios & DTMM & Benéfico \\
\hline Carlson et al (2001) $12 * * *$ & 44 & $\begin{array}{l}\text { Treino da respiração, relaxamento e } \\
\text { reeducação postural, placa e autocuidado }\end{array}$ & DOF & $\begin{array}{l}\text { Benéfico a curto e longo } \\
\text { prazos }\end{array}$ \\
\hline Grace et al $(2002)^{30 * *}$ & 45 & $\begin{array}{l}\text { Placa, orientação, medicação, exercícios } \\
\text { domiciliares, termoterapia, uso do BAE }\end{array}$ & DTM & Benéfico \\
\hline Stiesch-Scholz et al $(2002)^{32 * *}$ & 72 & $\begin{array}{l}\text { Placa e tratamento médico, fisioterapia, } \\
\text { exercícios e terapia manual }\end{array}$ & DDSR & Benéfico \\
\hline Dworkin et al (2002) $44 * * *$ & 117 & $\begin{array}{l}\text { Terapia cognitiva comportamental, gelo, } \\
\text { orientações, medicação, placa }\end{array}$ & DTM & Benéfico \\
\hline Magnusson et al (2002) $11 * * *$ & 135 & Placa isolada e com exercícios terapêuticos & DTM & Benéfico a longo prazo \\
\hline Dworkin et al (2002) $54 * * *$ & 124 & $\begin{array}{l}\text { Terapia comportamental, exercícios, } \\
\text { alongamento, compressa quente, gelo, } \\
\text { orientações, medicação, placa }\end{array}$ & DOF & Indefinidos \\
\hline $\begin{array}{l}\text { Anastassaki \& Magnusson (2004) } \\
56 * * *\end{array}$ & 3194 & $\begin{array}{l}\text { Conservador: placa, medicamentos, exer- } \\
\text { cícios mandibulares, acupuntura, outras }\end{array}$ & DTM & $\begin{array}{l}\text { Benéfico a DDSR, DTMA e } \\
\text { DTMM; pobre para DOF }\end{array}$ \\
\hline Babadag et al (2004) 33 & 25 & Analgésicos, placa de oclusão na ATM & DDCR & Benéfico \\
\hline Yoda et al $(2006)^{31 * *}$ & 104 & Exercício e placa & DDSR & Benéfico \\
\hline Truelove $(2006)^{13 *}$ & 200 & $\begin{array}{l}\text { Conservador: orientações, autocuidado e } \\
\text { dispositivo intra-oral }\end{array}$ & DTM & Benéfico \\
\hline
\end{tabular}

Tipo de estudo: * randomizado; **critérios de inclusão/exclusão; ${ }^{* * *}$ randomizado + critérios; N = número de sujeitos no estudo; DTM = disfunção temporomandibular; DTMM = disfunção temporomandibular miogênica; DTMA = disfunção temporomandibular artrogênica; DDSR = disfunção de disco sem redução; DDCR = disfunção de disco com redução; DOF = dor orofacial; ATM = articulação

temporomandibular 
atividades de vida diária pode auxiliar significativamente no controle da sintomatologia da DTM ${ }^{1,38}$. As orientações associadas a exercícios terapêuticos e à terapia manual podem ser eficazes no tratamento de pacientes com deslocamento de disco, mesmo sem haver o reposicionamento ${ }^{39,40}$. Os exercícios domiciliares podem ser benéficos para os que não melhoram somente com o tratamento convencional ${ }^{41}$.

Michelotti et al. ${ }^{15}$ compararam dois tratamentos e após três meses constataram que o grupo que recebeu somente orientação obteve $57 \%$ de melhora e o que combinou exercícios domiciliares à orientação, 77\% de melhora. Pacientes que fizeram fisioterapia e praticaram regularmente autocuidado obtiveram sucesso no relaxamento da musculatura mastigatória, alívio da dor e melhora nos sintomas da depressão e na qualidade do sono. As orientações de autocuidado, o esclarecimento dos fatores de risco e o treinamento de exercícios domiciliares proporcionam ganhos de ordem psicológica, pois diminuem a ansiedade ${ }^{42-44}$.

\section{A ssociado a reeducação postural, terapia manual e relaxamento}

A associação de exercícios terapêuticos e reeducação da postura foi mais eficaz do que o relaxamento ${ }^{45}$ e orientação de autocuidado ${ }^{37}$. Estudos ${ }^{12,46-49} \mathrm{em}$ que se associaram reeducação postural por meio de exercícios terapêuticos, terapia manual e relaxamento concluem que esses procedimentos parecem ser úteis nos casos de deslocamento anterior do disco com redução, síndrome dolorosa miofascial e DTM miogênica. Augustine et al. ${ }^{50}$ sugerem que a DTM pode estar associada à anteriorização da cabeça e que alongamentos e exercícios podem contribuir para correções posturais e redução dos sintomas.
Sherman et al. ${ }^{51}$ analisaram o efeito do relaxamento muscular em pacientes com dor orofacial. Os resultados indicaram aumento da imunoglobulina $A$ na saliva, sugerindo que este pode ser outro potencial benefício do treinamento progressivo em indivíduos com dor crônica.

Alguns estudos relatam o benefício dos exercícios terapêuticos e terapia manual, acrescidos de eletroterapia, nas DTMs miogênicas e artrogênicas ${ }^{1,14,21,52}$; e Machado \& Lima ${ }^{53}$ descrevem um estudo de caso em que, além da conduta odontológica, realizou-se manobra osteopática da coluna cervical, tendo como resultado redução do quadro álgico.

Apesar dos resultados favoráveis encontrados na literatura, um fator limitante nos estudos sobre terapia manual é a falta de descrição minuciosa das técnicas utilizadas, tendo em vista que os termos terapia manual, mobilização e manipulação são genéricos e podem incluir muitas formas de movimento passivo.

Quadro 2 Estudos em que se utilizaram exercícios associados a outros procedimentos

\begin{tabular}{|c|c|c|c|}
\hline Autoria, ano, tipo de estudo & $\mathrm{N}$ & Tratamento proposto & Disfunção Resultados \\
\hline Carlson et al (1991)45*** & 34 & Reeducação postural, alongamento & DTMM Benéfico \\
\hline Komiyama et al (1999)39*** & 60 & Intervenção cognitiva e correção postural & DTMM Benéfico \\
\hline Roychoudhury et al (1999)16** & 50 & Cirurgia & DTMA Benéfico \\
\hline Wright et al $(2000)^{37 * * *}$ & 60 & Reeducação postural e orientações & DTM Benéfico \\
\hline Nicolakis et al $(2000) 46 * * *$ & 30 & Reeducação postural e relaxamento & DTMA Benéfico \\
\hline Yuasa e Kurita(2001) $37 * * *$ & 60 & Antiinflamatório e fisioterapia & DDSR Benéfico \\
\hline Nicolakis et al $(2001)^{47 * * *}$ & 20 & $\begin{array}{l}\text { Terapia manual, correção postural, } \\
\text { relaxamento e orientações }\end{array}$ & DTMA Benéfico na osteoartrose \\
\hline Nicolakis et al (2001)48*** & 20 & $\begin{array}{l}\text { Terapia manual, reeducação postural, } \\
\text { relaxamento e orientações }\end{array}$ & DDSR Benéfico \\
\hline Nicolakis et al (2002) $49 * * *$ & 20 & Reeducação postural, relaxamento, orientação & DTMM Benéfico \\
\hline Delaat et al (2003) $14 * *$ & 26 & Massagem, ultra-som, alongamento,orientação & DTMM Benéfico \\
\hline Matta e Honorato (2003) $1 * *$ & 46 & Orientações e correção postural & DTM Benéfico \\
\hline Yoda et al $(2003)^{39 * * *}$ & 42 & Exercícios & DDSR Benéfico \\
\hline Rohling et al $(2003)^{55 * *}$ & 30 & $\begin{array}{l}\text { Ultra-som, crioterapia, alongamento, } \\
\text { acupuntura }\end{array}$ & DTMM Benéfico \\
\hline Michelotti et al (2004) $15 *$ & 70 & $\begin{array}{l}\text { Orientação de autocuidado e exercícios } \\
\text { domiciliares }\end{array}$ & DTMM Benéfico \\
\hline Riley et al (2007)43 & 126 & $\begin{array}{l}\text { Autocuidado, repouso, relaxamento, } \\
\text { massagem, exercícios, medicamentos }\end{array}$ & DOF Benéfico na depressão e sono \\
\hline Sato e Kawamura (2007)5 & 23 & Exercícios de abertura bucal & DDSR Sem melhora \\
\hline Wright $(2007)^{59}$ & 200 & Fisioterapia & DTM Benéfico \\
\hline Augustine et al (2008)50 & 29 & Alongamento e exercícios & DTM Benéfico \\
\hline Katsoulis e Richter (2008) 42 & 27 & Fisioterapia e autocuidado & DTMM Benéfico \\
\hline
\end{tabular}


Quadro 3 Estudos de caso em que se utilizaram exercícios

\begin{tabular}{|llll|}
\hline Autoria, ano & Tratamento proposto & Disfunção Resultados \\
\hline Waide et al (1992)52 & Estimulação elétrica, TENS, compressa quente, ultra-som, mobilização, gelo & DTM & Benéfico \\
\hline Zeno et al (2001)41 & Exercícios domiciliares & DTMA & Benéfico \\
\hline Machado e Lima (2004) ${ }^{53}$ & $\begin{array}{l}\text { Exercícios, terapia manual, alongamento passivo, manipulação cervical, } \\
\text { manobra osteopática }\end{array}$ & DOF & Benéfico \\
\hline Cleland e Palmer (2004)40 & Terapia manual, exercício terapêutico e orientações & DDSR & Benéfico \\
\hline Babu et al (2008)6 & Ultra-som, mobilização, relaxamento, exercícios, bandagem & DTM Sem melhora \\
\hline
\end{tabular}

DTM = disfunção temporomandibular; DTMA = disfunção temporomandibular artrogênica; DOF = dor orofacial; DDSR = disfunção de disco sem redução

\section{A ssociado a eletroterapia, acupuntura e crioterapia}

A associação de exercícios terapêuticos e eletroterapia tem se mostrado eficaz no tratamento da DTM miogênica $^{1,14,21,52,54-56}$ e no pós-cirúrgico ${ }^{52}$, resultando na normalização da amplitude de movimento mandibular, eliminação da dor e da inflamação e função mandibular sem restrição. $\mathrm{O}$ uso do gelo associado a outras modalidades de tratamento melhora a mobilidade mandibular, elimina a dor e a inflamação ${ }^{52}$ e, associado ao alongamento, pode diminuir a dor à palpação, nos casos de dor miofascial ${ }^{55}$. Já a acupuntura associada ao alongamento demonstrou melhora da remissão da dor espontânea e à palpação, nos casos de dor miofascial ${ }^{27,55}$. No entanto, na dor orofacial inespecífica, o resultado foi menos satisfatório ${ }^{57}$.

Michelotti et al. ${ }^{58}$, ao verificar a eficácia dos exercícios domiciliares na DTM miogênica, não chegaram a resultados conclusivos, argumentando pela necessidade de mais estudos clínicos randomizados.

\section{N ão associado, utilizado isoladamente}

$\mathrm{O}$ alongamento no tratamento da DTM miogênica teve como resultado redução da atividade eletromiográfica dos músculos da mastigação ${ }^{45}$. Os exercícios terapêuticos podem contribuir na redução efetiva do estalo em adultos com deslocamento de disco ${ }^{39}$ e os exercícios de abertura bucal no pósoperatório foram eficazes no tratamento a longo prazo ${ }^{16}$. O sucesso no tratamento é representado pela redução da dor, melhora funcional e da qualidade de vida; alguns autores referem melhora dos aspectos psicológicos e da mobilidade, não importando o tipo de exercício usado. É ressaltada a importância da boa orientação e motivação para os exercícios domiciliares, visto que a condição crônica impõe um cuidado constan$\mathrm{te}^{8,12,54,57}$. O tratamento conservador de fisioterapia foi capaz de provocar uma redução nos sintomas e promover alívio nas patologias relacionadas ao sistema auditivo ${ }^{59}$.

\section{CONCLUSÄO}

Nos ensaios clínicos aqui examinados os exercícios terapêuticos foram empregados de forma combinada a outros recursos de fisioterapia (terapia manual, termoterapia, fototerapia, eletroterapia e reeducação postural), ou integrada à odontologia, o que impede a verificação clara da contribuição dos exercícios terapêuticos.

Os ganhos obtidos com o tratamento tendem a se manter a curto e longo prazo, sobretudo quando o paciente recebe orientações de autocuidado e treinamento de exercícios domiciliares. Os estudos concluem que a aplicação de exercícios terapêuticos pode contribuir para o tratamento da DTM, porém mais estudos com metodologia mais padronizada devem ser estimulados. 


\section{REFERÊNCIAS}

1 Matta MA, Honorato DC. Uma abordagem fisioterapêutica nas desordens temporomandibulares: estudo restrospectivo. Rev Fisioter Univ São Paulo. 2003;10(2):77-83.

2 Okeson, JP. Fundamentos da oclusão e DTM. São Paulo: Artes Médicas; 1998.

3 Grossi DB, Guirro R, Costa EP, Arthuri MT. Proposta de uma ficha de avaliação para desordem craniomandibular a partir da caracterização dos pacientes atendidos na Clinica de Fisioterapia da Unimep. Rev Fisioter Univ São Paulo. 2001;8(1):30-9.

4 Biasotto-Gonzalez DA. Abordagem interdisciplinar das disfunções temporomandibulares. São Paulo: Manole; 2005.

5 Sato S, Kawamura H. Evaluation of mouth opening exercise after pumping of the temporomandibular joint in patients with nonreducing disc displacement. J Oral Maxillofac Surg. 2008;66(3):436-40.

6 Babu AS, John SM, Unni A. Strapping for temporomandibular joint dysfunction. Indian J Dent Res. 2008;19(3):278-9.

7 Widmark G, Haraldsson T, Kahnberg KE. Effects of conservative treatment in patients who later will be candidates for TMJ surgery. Swed Dent J. 1994;18(4):139-47.

8 Krogstad BS, Jokstad A, Dahl BL, Vassend O. Relationships between risk factors and treatment outcome in a group of patients with temporomandibular disorders. J Orofac Pain. 1996;10(1):48-53.

9 Marzola FT, Marques AP, Marzola C. Contribuiç̧ão da Fisioterapia para a Odontologia nas disfunções da articulação temporomandibular. Rev Odontol Cienc Fac Odontol PUC-RS 2002;17(36):119-34.

10 Fricton JR. Management of masticatory myofascial pain. Semin Orthod. 1995;1(4):229-43.

11 Magnusson T, Egermark I, Carlsson GE. Treatment received, treatment demand, and treatment need for temporomandibular disorders in 35-year-old subjects. Cranio. 2002;20(1):11-7.

12 Carlson CR, Bertrand PM, Ehrlich AD, Maxwell AW, Burton RG. Physical self-regulation training for the management of temporomandibular disorders. J Orofac Pain. 2001;15(1):47-55.

13 Truelove E, Huggins KH, Mancl L, Dworkin SF. The efficacy of tradicional, low-cost and nonsplint therapies for temporomandibular disorder: a randomized controlled trial. JADA. 2006;137(8):1099-107.
14 De Laat A, Stappaerts K, Papy S. Counseling and physical therapy as treatment for myofascial pan of masticatory system. J Orofac Pain. 2003;17(1):42-9.

15 Michelotti A, Steenks MH, Farella M, Parisini F, Cimino $R$, Martina $R$. The additional value of a home physical therapy refimen versus patient education only for the treatment of myofascial pain of the jaw muscles: shortterm results of a randomized clinical trial. J Orofac Pain. 2004;18(2):114-25.

16 Roychoudhury ABSD, Parkash HMDS, Trikha ADA. Function restoration by gap arthroplasty in temporomandibular joint ankylosis: a report of 50 cases. Oral Surg Oral Med Oral Pathol Oral Radiol Endod. 1999; 87(2):166-9.

17 Porto FR. Atuação fisioterapêutica nas desordens temporomandibulares. Rev Serv ATM. 2002;2(2):67-9.

18 Medlicott MS, Harris SR. A systematic review of the effectiveness of exercise, manual therapy, electrotherapy, relaxation training, and biofeedback in the management of temporomandibular disorder. Phys Ther. 2006;86(7):955-73.

19 Bruce S. Management of internal derangements of temporomandibular joint. Semin Orthodont. 1995; 1(4):244-57.

20 Tedeshi F, Marques AP. O papel da fisioterapia nas disfunções temporomandibulares. Rev Fisioter Univ São Paulo. 1999;6(2):172.

21 Feine JS, Widmer CG, Lund JP. Physical therapy: a critique. Oral Surg Oral Med Oral Pathol Oral Radiol Endod. 1997;83(1)123-7.

22 Fricton J. Myogenous temporomandibular disorders: diagnostic and management considerations. Dent Clin North Am. 2007;51(1):61-83.

23 McNeely ML, Armijo Olivo S, Magee DJ. A systematic review of the effectiveness of physical therapy interventions for temporomandibular disorders. Phys Ther. 2006;86(5):710-25.

24 Bessa-Nogueira RV, Vasconcelos BC, Niederman R. The methodological quality of systematic reviews comparing temporomandibular joint disorder surgical and nonsurgical treatment. BMC Oral Health. 2008;26(8):1-10.

25 Mannheimer JS. Limited evidence to support the use of physical therapy for temporomandibular disorder. Evid Based Dent. 2007;8(4):110-1.

26 Felício CM, Rodrigues da Silva MA, Mazzetto MO, Centola AL. Myofunctional therapy combined with occlusal splint in treatment of temporomandibular joint dysfunction - pain syndrome. Braz Dent J. 1991;2(1):27-33. 


\section{R eferências (cont.)}

27 Magnusson T, Syrén M. Therapeutic jaw exercises and interoclusal appliance therapy. A comparison between two common treatments of temporomandibular disorders. Swed Dent J 1999;23(1):27-37.

28 Krogstad BS, Jokstad A, Dahl BL, Soboleva U. Somatic complaints, psychologic distress, and treatment outcome in two groups of TMD patients, one previously subjected to whiplash injury. J Orofac Pain. 1998;12(2):136-44.

29 McNamara JA, Seligman DA, Okeson JP. Occlusion, Orthodontic treatment, and temoromandibular disorders: a review. J Orofac Pain. 1995;9(1):73-90.

30 Grace EG, Sarlani E, Reid B. The use of an oral exercise device in the treatment of muscular TMD. Cranio. 2002;20(3):204-8.

31 Yoda T, Sakamoto I, Imai H, Ohashi K, Hoshi K, Kusama $M$, et al. Response of temporomandibular joint intermittent closed lock to different treatment modalities: a multicenter survey. Cranio. 2006;24(2):130-6.

32 Stiesch-Scholz M, Fink M, Tschernitschek H, Rossbach A. Medical and physical therapy of temporomandibular joint disk displacement without reduction. Cranio. 2002;20(2):85-90.

33 Babadag M, Sahin M, Gorgun S. Pre and posttreatment analysis of clinical symptoms of patients with temporomandibular disease. Quintessence Int. 2004;35(10):811-4.

34 Murphy G. Physical medicine modalities and trigger point injections in the management of temporomandibular disorders and assessing treatment outcome. Oral Surg Oral Med Oral Pathol Oral Radiol Endod. 1997;83(1):118-22.

35 Brown DT, Gaudet EL Jr. Temporomandibular disorder treatment outcomes: second repot of large-scale prospective clinical study. Cranio. 2002; 20(4):244-53.

36 Al-Ani Z, Gray RJ, Davies SJ, Sloan P, Glenny AM. Stabilization splint therapy for the treatment of temporomandibular miofascial pain: a systematic review. J Dent Educ. 2005;69(11):1242-50.

37 Wright EF, Domenech MA, Fischer JR Jr. Usefulness of posture training for patients with temporomandibular disorders. J Am Dent Assoc. 2000;131(2):202-10.

38 Komiyama O, Kawara M, Arai M, Asano T, Kobayashi K. Posture correction as part of behavioural therapy in treatment of myofascial pain with limited opening. J Oral Rehabil. 1999;26(5):428-35.

39 Yoda T, Sakamoto I, Imai H, Honma Y, Shinjo Y, Takano A, et al. A randomized controlled trial of terapeutic exercice for clickink due to disc anterior displacement with reduction in temporomandibular joint. Cranio. 2003;21(1):10-6.
40 Cleland J, Palmer J. Effectiveness of manual physical therapy, therapeutic exercise, and patient education on bilateral disc displacement without reduction- of the temporomandibular joint: a single-case design. J Orthop Sports Phys Ther. 2004;34(9):535-48.

41 Zeno E, Griffin J, Boyd C, Oladehin A, Kasser R. The effects of a home exercise program on pain and perceived dysfunction in a woman with TMD: a case study. Cranio. 2001;19(4): 279-88.

42 Katsoulis J, Richter M. Efficacy of specific physiotherapy for temporomandibular joint dysfunction of muscular origin. Rev Stomatol Chir Maxillofac. 2008;109(1):9-14

43 Riley JL 3rd, Myers CD, Currie TP, Mayoral O, Harris RG, Fisher JA, et al. Self-care behaviors associated with myofascial temporomandibular disorder pain. J Orofac Pain. 2007;21(3):194-202.

44 Dworkin SF, Turner JA, Mancl L, Wilson L, Massoth D, Huggins $\mathrm{KH}$ et al. A randomized clinical trial of a tailored comprehensive care treatment program for temporomandibular disorders. J Orofac Pain. 2002;16(4):259-76.

45 Carlson CR, Okeson JP, Falace DA, Nitz AJ, Anderson D. Stretch-based relaxation and the reduction of EMG activity among masticatory muscle pain patients. J Craniomandib Disord. 1991;5(3):205-12.

46 Nicolakis P, Erdogmus B, Kopf A, Djaber-Ansari A, Piehslinger E, Fialka-Moser V. Exercise therapy for craniomandibular disorders. Arch Phys Med Rehabil. 2000;81(9):1137-42.

47 Nicolakis P, Burak EC, Kollmitzer J, Kopf A, Piehslinger E, Wiesinger GF, Fialka-Moser V. An investigation of the effectiveness of exercise and manual therapy in treating symptoms of TMJ osteoarthritis. Cranio. 2001;19(1):26-32.

48 Nicolakis P, Erdogmus B, Kopf A, Ebenbichler G, Kollmitzer J, Piehslinger E, Fialka-Moser V. Effectiveness of exercise therapy in patients with internal derangement of temporomandibular joint. J Oral Rehabil. 2001;28(12):1158-64.

49 Nicolakis P, Erdogmus B, Kopf A, Nicolakis M, Piehslinger E, Fialka-Moser V. Effectiveness of exercise therapy in patients with myofascial pain dysfunction syndrome. J Oral Rehabil. 2002;29(4):362-8.

50 Augustine C, Makofsky HW, Britt C, Adomsky B, Deshler JM, Ramirez P, Douris P. Use of the occivator for the correction of forward head posture, and implications for temporomandibular disorders: a pilot study. Cranio. 2008;26(2):136-43. 


\section{R eferências (cont.)}

51 Sherman JJ, Carlson CR, McCubbin JA, Wilson JF. Effects of stretch-based progressive relaxation training on the secretion of salivary immunoglobulin $A$ in orofacial pain patients. J Orofac Pain. 1997;11(2):155-24.

52 Waide FL, Bade DM, Lovasko J, Montana J. Clinical management of a patient following temporomandibular joint arthroscopy. Phys Ther. 1992;72(5):355-64.

53 Machado MR, Lima RHM. Abordagem fisioterápica no tratamento de desordem temporomandibular associada à protusão de cabeça: relato de caso clínico. Rev Serv ATM. 2004;4(2):40-4.

54 Dworkin SF, Huggins KH, Wilson L, Mancl L, Turner JA, Massoth $\mathrm{D}$ et al. A randomized clinical trial using research diagnostic criteria for temporomandibular disorders-axis II to target clinic cases for a tailored selfcare TMD treatment program. J Orofac Pain. 2002;16(1):48-63.
55 Rohling D, Mello EB, Porto FR. Estudo comparativo de alternativas terapêuticas em trigger points miofasciais. Rev Serv ATM. 2003;3(3):11-17.

56 Anastassaki A, Magnusson T. Patients referred to a specialist clinic because of suspected temporomandibular disorders: a survey of 3194 patients in respect of diagnoses, treatments, and treatment outcome. Acta Odontol Scand. 2004;62(4):183-92.

57 Krogstad BS, Jokstad A, Dahl BL, Soboleva U. Somatic complaints, psychologic distress, and treatment outcome in two groups of TMD patients, one previously subjected to whiplash injury. J Orofac Pain. 1998;12(2):136-44.

58 Michelotti A, Wijer A, Steenks M, Farella M. Homeexercise regimes for the management of non-specific temporomandibular disorders. J Oral Rehabil. 2005;32(11):779-85.

59 Wright EF. Otologic symptom improvement through TDM therapy. Quintessence Int. 2007;38(9):564-71. 